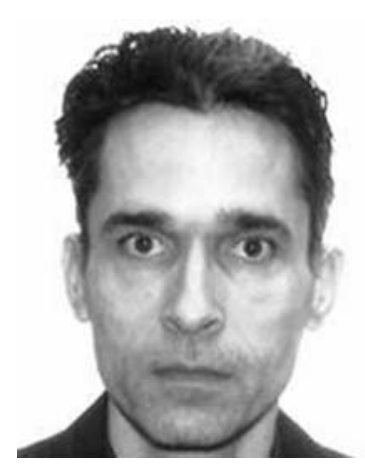

\title{
A Conversation with Alexandre Pouget
}

\author{
INTERVIEWER: CHARVy NARAIN \\ Former Senior Editor, Nature Neuroscience
}

Alexandre Pouget is a Professor in the Department of Basic Neuroscience at the University of Geneva.

Charvy Narain: You'll be talking about how the brain actually encodes information but from an abstract computational perspective. Are you comparing the brain to how a computer works? Is that the sort of approach that you're using?

Dr. Pouget: Yes, there is an interesting kind of analogy that you can draw there. Basically, with any kind of computing device, the first thing you need to figure out is how the device encodes information. If you want to understand how a computer works, you've got to understand first that it encodes information through those strings of bits, binary elements that can be 0 s or $1 \mathrm{~s}$. Unless you understand that, there is no way you can then understand how it actually computes.

Charvy Narain: How do you approach this problem with respect to the brain?

Dr. Pouget: We have to rely extensively on recordings from our colleagues who are doing experiments in animals and presenting a variety of stimuli. We're asking the animal to move and the same time we record from the neurons. Then we try to establish what the mapping is between the stimulus and the responses.

Charvy Narain: When you establish these sorts of mappings, what do you find?

Dr. Pouget: There are quite a few findings, but the main hypothesis we're pursuing is that the code in the brain must be redundant.

Modern computers can encode billions and billions of numbers with absolute reliability and no errors.

This precision comes with two different costs. One is power. We use about a volt to encode a bit. As a result, you need a lot of power for your computers. A supercomputer may cost $\$ 2$ million a year just in electricity.

The second issue is reliability. If any piece of hardware breaks in those computers so that the bits can no longer be encoded, you throw away the whole thing or at least, whatever board broke. So a computer is expensive to run and easy to damage, and the contrast with the brain is striking. It's exactly the opposite. In the U.S., you need only a few thousand dollars per year to feed your brain, and it is incredibly good at handling damage. At this point in my life, unfortunately, I lose something in the order of 30,000 neurons per day, and that's only going to get worse. Yet I'm told I continue to function reasonably well. In humans this is called graceful degradations. By the time you're 80 , you'll probably have half the brain you had when you were younger, and yet you will continue to function.

Charvy Narain: Even then, the human brain is handling far more data than any supercomputer.

Dr. Pouget: So one of the big questions for us is what's so special about the neural code that allows our brains to have these properties.

We think the key is redundancy. That is, instead of having one bit that's infinitely reliable, you have multiple copies of the same bit that don't have to be perfectly reliable. If you have that, then you can know the value of a bit not just by looking at one of them but by taking the average of the multiple copies that you have. Having multiple copies is what we call the redundant code.

Charvy Narain: Wouldn't you then need a lot of space? The brain is quite compact. If you have multiple bits of information, does that not create space constraints?

Dr. Pouget: It does. You end up having to have more neurons than you would if you were more efficient. But if you want to be robust to damage, you must be able to get the system to function reasonably well even when you've lost half the components in the system. That's the way our brain works. We possibly have way more neurons than we need in principle but this system needs much less energy, which is really important. It takes about a volt to encode a bit in a computer whereas the brain functions on millivolts, a thousand times less. 
So there are costs to everything. There's no free lunch, but nature seems to have gone for the solution of redundancy, with lots of neurons that are robust to damage.

Charvy Narain: What predictions emerge from this theory?

Dr. Pouget: Whenever you have a redundant code, one of its properties is that the neurons that all encode the same information should share information. We call that being "correlated."

They have to be correlated in a very specific way. It becomes a bit technical, but that's where our work is focusing. We have a very specific prediction about how the neuron should be correlated. We figured out the mathematics behind that about 4 years ago but were badly surprised when the data didn't match the prediction.

Dr. Pouget: People had measured correlation long before we did the mathematical work. When the correlation was first measured, people declared, "Oh, this correlation implies that the brain has a redundant code."

About 10 years ago, another group concluded that from what we can measure in the brain, it doesn't look like a redundant code.

Then we came along and said, "Yes, that's right. For a redundant code, we need another pattern of correlations." The data were already published, and it didn't fit our model, so we had a real puzzle in front of us.

Charvy Narain: How did you square the two?

Dr. Pouget: I'm a theoretician, so I love the data to be wrong, but the data are the data. What we came to realize is that the correlation we see in real neurons is probably a mixture of different factors. Some of them really constrain the code and make it redundant, while others are there because of the way the system is built.

We now have very good reason to believe that the measurements are completely dominated by the correlations that do not constrain the codes. For example, you may have different states of awareness that go up and down.

We know today that this kind of fluctuation in the overall activity of the brain creates a particular pattern of correlations. Those turn out to have almost no impact on the code, but it creates a large correlation that measures between 0 and 1 .

Our theoretical prediction is the correlation is about $0.01,10$ times smaller than the correlation that people measure.

Dr. Pouget: Correlations are notoriously difficult to measure. In neuroscience, if we can get about 10 trials, 20 trials, it's a lot and that's hard to get from animals. That's just not enough to find out the value of the correlation coefficient. We think that the correlations that we're looking for are buried under a lot of irrelevant fluctuation in the correlation coefficients.

Charvy Narain: So the correlation you're looking for is much tinier than these and being washed out by irrelevant correlations. Is there any way to get at the correlations you're interested in?

Dr. Pouget: We're not sure yet. It's going to be complicated but there is hope. Ultimately, what we're trying to do is to understand how neural activity controls behavior. That's really our final goal.

The way we do this nowadays is to quantify how much information is captured in your behavior about the stimulus that is making you react, or making you behave. This we can quantify very precisely.

We try to see if, in the brain, the information in different areas involved in behavior has the same order of magnitude as the behavior of your animal or of your subject. We try to match information.

What we want is not the correlations, which are boring, counterintuitive, and hard to measure, we want information. One way to do this is to measure the correlations and compute the information. Another way is to use techniques that bypass the correlation measure altogether and go straight for information.

The problem we've got in doing that is the need to measure all the neurons at the same time while an animal is behaving.

Dr. Pouget: Up until 10 years ago, all we could do was record one neuron in one animal while the animal is performing a task. We know there are millions of neurons involved in any particular task.

What's fantastic in our field is the recent explosion of technology. We are now able to record at least from 100, possibly 1000 neurons at the same time in a behaving animal. In fact, in some systems, we can record all of the neurons at once. We can finally see the entire system at work, live. We now have the techniques needed to try to figure out whether tiny correlations are there or not by measuring information.

Charvy Narain: What sort of techniques would allow you to record from many neurons at once?

Dr. Pouget: In the past, we recorded from neurons using electrical wires that push until they got very close to one neuron. Then we could hear the activity of that neuron and record its electrical activity.

Nowadays, there are stunning new techniques where dyes are put inside the cells. The dye can be expressed in all the cells at the same time. Those dyes are sensitive to calcium. Whenever a cell is active, sending signals, calcium gets inside for a short time, for 10 to 20 seconds.

The dyes expressed in those neurons can sense calcium and light up if they detect it. By looking at how much light comes out, we can figure out how much calcium gets into the cells and therefore how active they are.

Again, one has to keep in mind that we're measuring from cells that are one-tenth of the width of a hair and examining hundreds if not thousands of them at the same time.

Charvy Narain: What sort of work are you planning in the future?

Dr. Pouget: In the next few years, now that we have the technology, we can finally try to establish a precise map- 
ping between the population activity of all the neurons together while the animal is performing a task. We can try to understand what it is in the activity of those neurons that leads to the behavior.

When I was a grad student, 20 or 25 years ago that was the dream. I wasn't sure I would ever see a day where we would be able to do those experiments.
We're going to record all the neurons while the animal is performing the task, and we're going to use all the theoretical tools that we've developed while we were waiting for the technology to establish that mapping. It's going to be the first step toward understanding the mapping between neural activity or thoughts, or behavior, or desire or motivations. 


\section{$\$_{\text {CSH }}^{\infty}$ Cold Spring Harbor Symposia SYMPOSIA}

\section{A Conversation with Alexandre Pouget}

Cold Spring Harb Symp Quant Biol 2014 79: 285-287

Access the most recent version at doi:10.1101/sqb.2014.79.14

\section{License}

Email Alerting Receive free email alerts when new articles cite this article - sign up in Service the box at the top right corner of the article or click here. 